\title{
Optical tomography of Fock state superpositions
}

\author{
Sergey N. Filippov and Vladimir I. Man'ko
}

\author{
Moscow Institute of Physics and Technology (State University) \\ Institutskii per. 9, Dolgoprudnyi, Moscow Region 141700, Russia \\ and \\ P. N. Lebedev Physical Institute, Russian Academy of Sciences \\ Leninskii Prospect 53, Moscow 119991, Russia \\ e-mail: sergey.filippov@ phystech.edu, manko @ sci.lebedev.ru
}

\begin{abstract}
We consider optical tomography of photon Fock state superpositions in connection with recent experimental achievements. The emphasis is put on the fact that it suffices to represent the measured tomogram as a main result of the experiment. We suggest a test for checking the correctness of experimental data. Explicit expressions for optical tomograms of Fock state superpositions are given in terms of Hermite polynomials. Particular cases of vacuum and low photon-number state superposition are considered as well as influence of thermal noise on state purity is studied.
\end{abstract}

Keywords: optical tomogram, Fock state superpositions, tomographic-probability representation.

Last decades have seen a continuously growing interest to quantum phenomena. The research has become interdisciplinary and covered many fields from quantum optics and particle physics to quantum information processing and foundations of quantum mechanics. Preparation, evolution, and measurement of quantum states are focused on in numerous theoretical and experimental investigations. Also, a great effort was made in characterization of quantum state properties such as nonlocality and entanglement. Both explanation and prediction of experimental results resort to a mathematical description of quantum states. Dealing with quantum states of the electromagnetic field, widely used are the following descriptions: the density operator $\hat{\rho}$ and several quasidistribution functions like Wigner function. This is the Wigner function that is the main goal of many scientific laboratories interested in quantum state reconstruction. However, the probabilistic nature of quantum mechanics does not allow measuring Wigner function directly in the experiment. It can only be obtained by subsequent processing of data. In view of this fact, the question arises itself whether there exists such a probability description of quantum states which is accessible for a direct measuring. If such a description exists, then it can be considered as an advantageous notion of quantum states. Surprisingly, such a description does exist and it is an optical tomogram 1, 2. This means that there is no need to be aimed at obtaining Wigner function anymore.

Indeed, it was clarified recently 1,2 that any quantum state can be identified with the optical tomogram. From the mathematical point of view, the optical tomogram is nothing else but Radon transform of the Wigner function. Crucial point is that the Radon transform provides probability distributions (see, e.g. [3]) and such a distribution turns out to be measurable directly via the homodyne detection of photon states. The experimental output of such detection is exactly the optical tomogram. Originally, it was used [4,5] as a technical tool to reconstruct the Wigner function which is still usually identified with the photon quantum state. On the other hand, the optical tomogram contains complete information about all properties of a quantum state. In view of this fact, it is pointed out that the accent in homodyne detection experiments has to be put on the most accurate measuring of the optical tomogram. According to association "quantum state $\Longleftrightarrow$ measurable tomogram", it would be enough to present as a result of the experiment a plot of the tomogram.

Once optical tomogram is measured, there is a necessity to be aware of output data being indeed a tomogram of quantum state. In order to test correctness of the measured tomogram, one can utilize 
peculiar properties of quantum states. For example, one can check that the experimental results do not contradict to tomographic entropic inequalities [6], uncertainty relations [7] or purity constraints [8]. In other words, one must check that the tomogram fulfils specific requirements derived in [6] 9]. Apparently, these constraints are only necessary conditions but their violation serves as a direct evidence of either experiment or quantum theory being incorrect. In this paper, we will also present an additional necessary condition on optical tomogram and an estimation of the data accuracy.

The exciting progress of experimental techniques during last several years gave rise to creation of paradigmatic photon states like Fock state superpositions (FSS) $10-13]$. They are nothing else but dramatic representatives of nonclassical states [14]. The above discussion on optical tomogram as a primary notion of quantum states makes it reasonable to consider the optical tomography of FSS. The aim of our paper is to fill a gap between the experiment and the theoretical description of FSS. Namely, we obtain an explicit expression for the optical tomogram of FSS, analyze a highly simplified model of the influence of thermal noise on optical tomograms and purity of FSS. We also introduce a lower error bar on measured tomogram. The results of this consideration altogether with the outstanding techniques like those used in experiments 10 13, 15 17] could find application in further investigations for comparing output data with predicted ones, estimating purity and thermal noise presented, adjusting and calibrating the setup.

Optical tomogram of the state given by density operator $\hat{\rho}$ reads

$$
w(X, \theta)=\int G(X, \theta ; z, 0) G^{*}\left(X, \theta ; z^{\prime}, 0\right)\left\langle z|\hat{\rho}| z^{\prime}\right\rangle \mathrm{d} z \mathrm{~d} z^{\prime},
$$

where $G\left(q, t ; q^{\prime}, 0\right)$ is a conventional Green's function of the harmonic oscillator with Hamiltonian $\hat{H}=$ $\left(\hat{p}^{2}+\hat{q}^{2}\right) / 2=\left(\hat{a}^{\dagger} \hat{a}+1 / 2\right)$. Hereafter we use dimensionless units, namely, the Planck constant $\hbar=1$, the Boltzmann constant $k_{\mathrm{B}}=1$, etc. Formula (1) can be treated as a reconstruction of the quantum state of wave packet from position probability distributions measured during the packet's motion in the harmonic oscillator potential [18,19]. In case of optical tomography, the timelike evolution of the electromagnetic field is brought about by shifting the phase $\theta$ of the local oscillator. The tomogram $w(X, \theta)$ is the marginal distribution of the quadrature component $X$ of the electric field strength, with $X$ being rotated by angle $\theta$ in the quadrature phase space.

Let us consider the photon state of one-mode electromagnetic field $|\psi\rangle=\sum_{n=0}^{N} c_{n}|n\rangle$, where $|n\rangle$ is the photon Fock state, $N<\infty$. Density operator of such a pure state is

$$
\hat{\rho}=|\psi\rangle\left\langle\left.\psi\left|=\sum_{n=0}^{N}\right| c_{n}\right|^{2} \mid n\right\rangle\langle n|+\sum_{n<k}\left(c_{n}^{*} c_{k}|k\rangle\langle n|+\text { h.c. }\right) .
$$

The Green's function $G\left(q, t ; q^{\prime}, 0\right)$ determines an evolution of Hamiltonian eigenstates $|n\rangle$ as follows

$$
\int G\left(q, t ; q^{\prime}, 0\right)\left\langle q^{\prime} \mid n\right\rangle \mathrm{d} q^{\prime}=\langle q \mid n\rangle e^{-i(n+1 / 2) t}=\frac{1}{\sqrt{\sqrt{\pi} 2^{n} n !}} H_{n}(q) e^{-q^{2} / 2-i(n+1 / 2) t},
$$

where $H_{n}(q)$ is the Hermite polynomial of degree $n$. Substituting (2) and (3) in (1), we readily obtain the optical tomogram of Fock state superposition

$$
\begin{aligned}
w_{\mathrm{FSS}}(X, \theta) & =\frac{e^{-X^{2}}}{\sqrt{\pi}}\left[\sum_{n=0}^{N} \frac{\left|c_{n}\right|^{2}}{2^{n} n !} H_{n}^{2}(X)+\sum_{n<k} \frac{\left(c_{n}^{*} c_{k} e^{i(n-k) \theta}+\text { c.c. }\right)}{\sqrt{2^{n+k} n ! k !}} H_{n}(X) H_{k}(X)\right] \\
& =\frac{e^{-X^{2}}}{\sqrt{\pi}}\left[\sum_{n=0}^{N} \frac{\left|c_{n}\right|^{2}}{2^{n} n !} H_{n}^{2}(X)+\sum_{n<k} \frac{\left|c_{n}\right|\left|c_{k}\right| \cos \left((n-k) \theta-\left(\varphi_{n}-\varphi_{k}\right)\right)}{\sqrt{2^{n+k-2} n ! k !}} H_{n}(X) H_{k}(X)\right],
\end{aligned}
$$

where we have extracted phases from coefficients $c_{n}=\left|c_{n}\right| e^{i \varphi_{n}}$ and $c_{k}=\left|c_{k}\right| e^{i \varphi_{k}}$. 
(a)

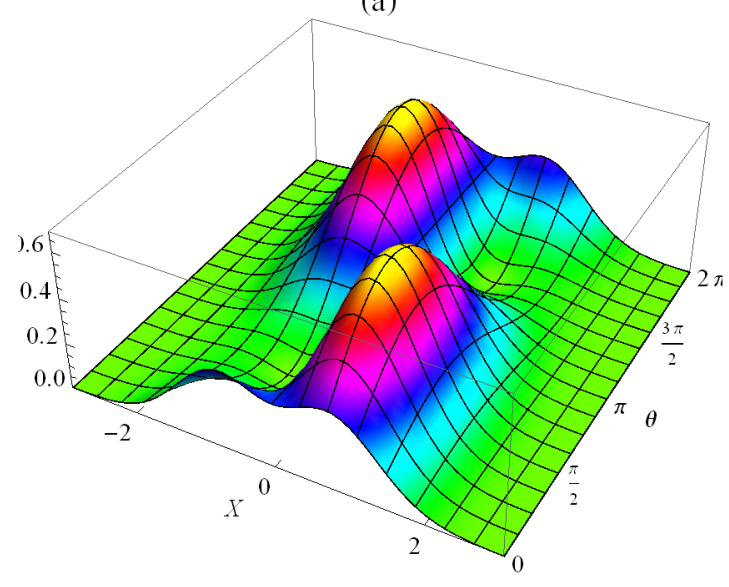

(c)

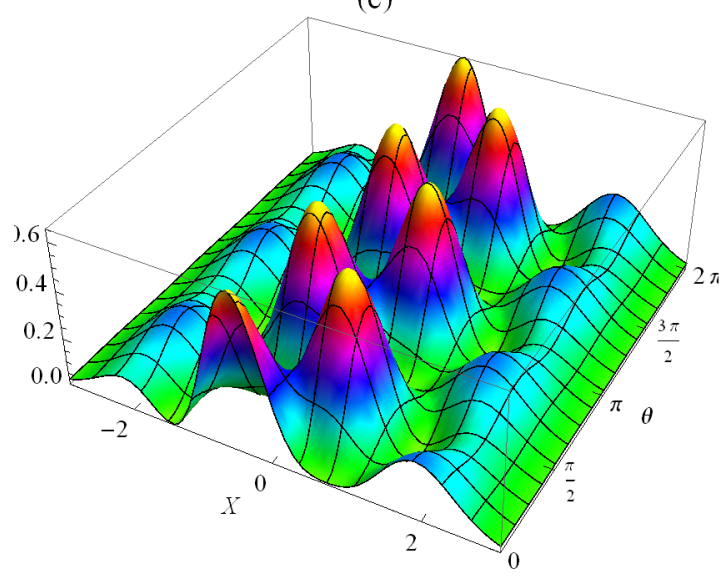

(b)

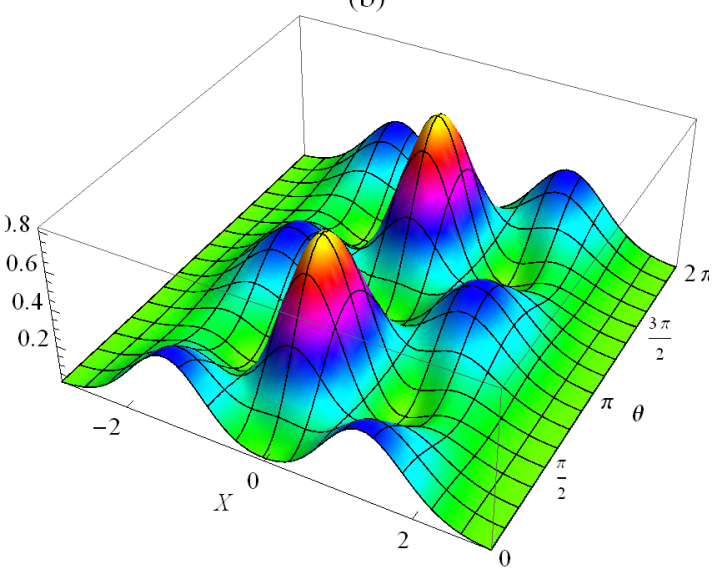

(d)

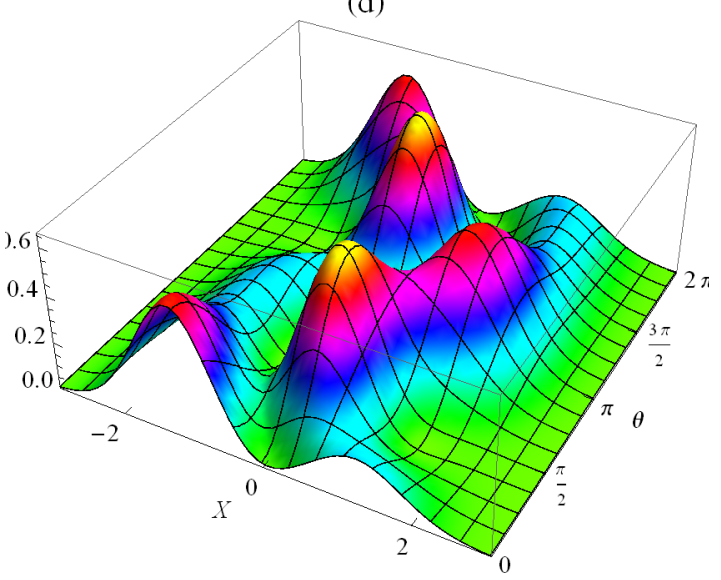

Figure 1: Optical tomograms $w(X, \theta)$ of Fock state superpositions: $(|0\rangle+i|1\rangle) / \sqrt{2}(\mathrm{a}),(|0\rangle+|2\rangle) / \sqrt{2}$ (b), $(|0\rangle+|3\rangle) / \sqrt{2}(\mathrm{c})$, and $\left(|0\rangle+e^{i 2 \pi / 3}|1\rangle+|2\rangle\right) / \sqrt{3}(\mathrm{~d})$.

Examples of FSS optical tomograms are illustrated in Fig. 1. It is readily observed that the tomograms satisfy the relation $w(X, \theta)=w(-X, \theta+\pi)$, which also follows from analytical consideration. This simple constraint on experimental data allows checking the accuracy of the measured tomogram $w^{\text {meas }}(X, \theta)$. Indeed, the difference $\left[w^{\text {meas }}(X, \theta)-w^{\text {meas }}(-X, \theta+\pi)\right]$ must be zero for an exactly measured tomogram. The deviation of this quantity from the zero level for all the local oscillator phases, e.g. $\sup _{X \in \mathbb{R}, \theta \in[0,2 \pi]}\left|w^{\text {meas }}(X, \theta)-w^{\text {meas }}(-X, \theta+\pi)\right|$, is suggested to use as an indicator of homodyne detection precision.

It is worth mentioning that the Pauli problem can be constructively solved for FSS [20]. In other words, for FSS it is sufficient to measure $w(X, \theta=0)$ and $w(X, \theta=\pi / 2)$ to find the initial state, up to the well-known twofold ambiguity. This result is based on the fact that we know a priori that the state of the system is pure and comprises a finite number of Fock states. However, in real experiments perfectly pure states are hardly achievable. For instance, optical tomography of microwave electromagnetic field exploits linear amplifiers which unavoidably introduce a noise. We will treat this noise as thermal one with the effective temperature $T$. We find it reasonable to consider the influence of such thermal noise on the purity of the desired FSS. Moreover, the purity can be directly calculated via measured optical tomogram [8] avoiding the density matrix formalism. Comparison of obtained and theoretically predicted 

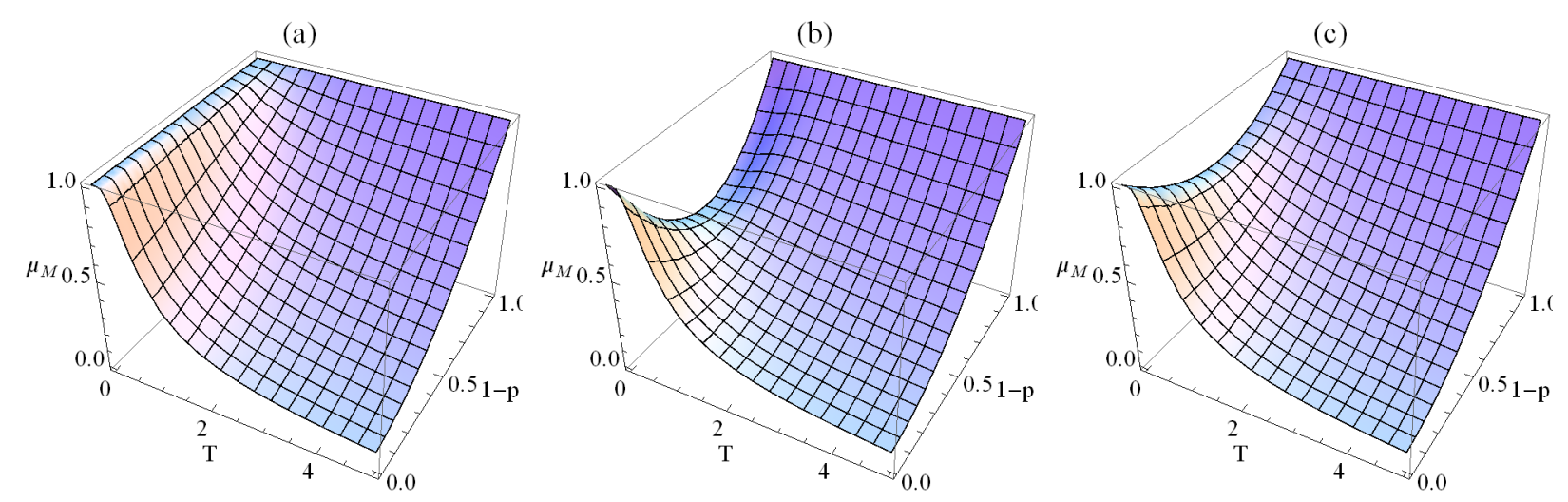

Figure 2: Purity $\mu_{\text {mix }}$ of mixture of Fock state superpositions with thermal noise vs. temperature $T$ and mixture coefficient $p$ : vacuum state $|0\rangle$ (a), one-photon state $|1\rangle(\mathrm{b})$, and $\left(|0\rangle+e^{i \varphi_{1}}|1\rangle\right) / \sqrt{2}$ (c).

values of purity can be used for testing the correctness of data, calibrating and adjusting the apparatus.

For the sake of simplicity, we consider the following model. The density operator of thermal noise has the form $\hat{\rho}_{\mathrm{th}}=Z^{-1} e^{-\hat{H} / T}$, where $Z=\operatorname{Tr}\left[e^{-\hat{H} / T}\right]=\frac{1}{2}\left(\sinh \frac{1}{2 T}\right)^{-1}$ and $T$ is a temperature. The associated optical tomogram reads $w_{\mathrm{T}}(X, \theta)=\left(\sqrt{2 \pi \sigma^{2}}\right)^{-1 / 2} e^{-X^{2} / 2 \sigma^{2}}$, where $\sigma^{2}=\frac{1}{2} \operatorname{coth} \frac{1}{2 T}$. Mixture of the superposition of Fock states (2) and thermal noise is given by density operator $\hat{\rho}_{\text {mix }}=(1-p) \hat{\rho}+p \hat{\rho}_{\text {th }}$, where the parameter $0 \leq p \leq 1$ shows how much noise is added. The corresponding optical tomogram reads $w_{\text {mix }}(X, \theta)=(1-p) w_{\mathrm{FSS}}(X, \theta)+p w_{\mathrm{th}}(X, \theta)$. Purity of this state is

$$
\begin{aligned}
\mu_{\text {mix }} & \equiv \operatorname{Tr}\left[\hat{\rho}_{\text {mix }}^{2}\right]=(1-p)^{2}+2(1-p) p\left\langle\psi\left|\hat{\rho}_{\mathrm{T}}\right| \psi\right\rangle+p^{2} \operatorname{Tr}\left[\hat{\rho}_{\mathrm{th}}^{2}\right] \\
& =(1-p)^{2}+4(1-p) p \sinh \frac{1}{2 T} \sum_{n=0}^{N}\left|c_{n}\right|^{2} e^{-\left(n+\frac{1}{2}\right) / T}+p^{2} \tanh \frac{1}{2 T}
\end{aligned}
$$

where we have taken into account the diagonal form of thermal noise $\hat{\rho}_{\mathrm{T}}$ in the basis of Fock states. Dependence of state purity on thermal noise is depicted for some examples in Fig. 2. If $T \rightarrow \infty$, then $\mu_{\text {mix }} \approx(1-p)^{2}+p^{2} / 2 T$. If $T \rightarrow 0$, then the thermal noise reduces to vacuum mode and its effect on purity depends on two factors: the noise strength $p$ and whether the vacuum state was included in the initial FSS. If vacuum state is absent in FSS, then $\mu_{\text {mix }}(T=0)$ is the same for all such FSS. It means that the parameter $p$ can be evaluated by one-photon state. It is worth mentioning a non-monotonic dependence of one-photon state purity on $T$ for a fixed $p<1 / 2$ because $\mu_{\text {mix }} \approx(1-p)^{2}+p^{2}+2 p(1-2 p) e^{-1 / T}$ if $T \ll 1$ (see Fig. 2b). In the paper [21], the effect of purity oscillations is discussed. In present work we note that the formula for purity in terms of optical tomograms can also be used to describe such phenomena.

To conclude, we summarize the main results of the paper. We studied the optical tomograms of Fock state superpositions in view of several experiments 10 13] devoted to measuring the homodyne quadrature distributions in some nonclassical photon states [14. We point out that the efforts of experiments should be focused on as precise measurement of optical tomograms as possible. The optical tomogram provides the complete information about a quantum state and the correctness of measured tomogram can be checked by a series of constraints, one of which is introduced in this paper. The other tests of the precision of homodyne detecting photon states can be also used 66 9. We emphasize that there is no need to convert the tomogram into Wigner function or other quasi-probability functions. Being applied to Fock state superpositions, the optical tomogram is obtained in explicit form (4). We have also 
analyzed the purity of a Fock state superposition mixed with a thermal noise unavoidably presented in experiments.

The authors appreciate the referees for useful remarks. The authors thank the Russian Foundation for Basic Research for partial support under Projects Nos. 09-02-00142 and 10-02-00312. S.N.F. thanks the Russian Science Support Foundation for support under Project "Best postgraduates of the Russian Academy of Sciences 2010" and the Ministry of Education and Science of the Russian Federation for support under Projects Nos. 2.1.1/5909, П558, and 14.740.11.0497.

\section{References}

[1] S. Mancini, V. I. Man'ko, and P. Tombesi, Phys. Lett. A, 213, 1 (1996).

[2] A. Ibort, V. I. Man'ko, G. Marmo, A. Simoni, and F. Ventriglia, Phys. Scr., 79, 065013 (2009).

[3] B. Militello, V. I. Man'ko, M. A. Man’ko, and A. Messina, Phys. Scr., 80, 058102 (2009).

[4] D. T. Smithey, M. Beck, M. G. Raymer, and A. Faridani, Phys. Rev. Lett., 70, 1244 (1993).

[5] A. I. Lvovsky and M. G. Raymer, Rev. Mod. Phys., 81, 299 (2009).

[6] S. De Nicola, R. Fedele, M. A. Man'ko, and V. I. Man'ko, Eur. Phys. J. B, 52, 191 (2006).

[7] V. I. Man'ko, G. Marmo, A. Simoni, and F. Ventriglia, Adv. Sci. Lett., 2, 517 (2009).

[8] V. I. Man'ko, G. Marmo, A. Porzio, S. Solimeno, and F. Ventriglia, "Homodyne extimation of quantum states purity by exploiting covariant uncertainty relation," arXiv:1012.3297v1 [quant-ph] (2010).

[9] M. A. Man'ko and V. I. Manko, Found. Phys., doi:10.1007/s10701-009-9403-9 (2009).

[10] M. Hofheinz, H. Wang, M. Ansmann, R. C. Bialczak, E. Lucero, M. Neeley, A. D. OConnell, D. Sank, J. Wenner, J. M. Martinis, and A. N. Cleland, Nature, 459, 546 (2009).

[11] E. Bimbard, N. Jain, A. MacRae, and A. I. Lvovsky, Nature Photonics, 4, 243 (2010).

[12] A. S. Parkins, P. Marte, P. Zoller, and H. J. Kimble, Fundamentals of Quantum Optics III, Lecture Notes in Physics, 420, 210 (1993).

[13] S. Deléglise, I. Dotsenko, C. Sayrin, J. Bernu, M. Brune, J.-M. Raimond, and S. Haroche, Nature, 455, 510 (2008).

[14] V. V. Dodonov, J. Opt. B: Quantum Semiclass. Opt., 4, R1, (2002).

[15] A. Ourjoumtsev, R. Tualle-Brouri, J. Laurat, and P. Grangier, Science, 312, 83 (2006).

[16] A. Zavatta, V. Parigi, M. S. Kim, H. Jeong, and M. Bellini, Phys. Rev. Lett., 103, 140406 (2009).

[17] C. Eichler, D. Bozyigit, C. Lang, M. Baur, L. Steffen, J. M. Fink, S. Filipp, and A. Wallraff, "Photon state tomography for two-mode correlated itinerant microwave fields," arXiv:1101.2136v1 [quant-ph] (2011).

[18] U. Leonhardt and M. G. Raymer, Phys. Rev. Lett., 76, 1985 (1996).

[19] A. del Campo, V. I. Man'ko, and G. Marmo, Phys. Rev. A, 78, 025602 (2008).

[20] A. Orłowski and H. Paul, Phys. Rev. A, 50, R921 (1994).

[21] B. Militello, K. Yuasa, H. Nakazato, and A. Messina, Phys. Rev. A, 77, 042109 (2008). 